\title{
AUS DEM VORWORT ZUR ERSTEN AUFLAGE
}

Der Aufbau und Inhalt des vorliegenden Bandes ,Quanten“" wird in der Einleitung ausführlich besprochen. Er enthält sieben Teile, deren Inhalt eng zusammenhängt, so da $\beta$ sich für das Studium der einzelnen Teile eine gewisse Reihenfolge empfiehlt, die durch das untenstehende Pfeilschema angedeutet wird. Teil 2 über die Teilchenquantelung bereitet dem Anfänger gewisse Schwierigkeiten. Dafür wurde Teil 3 so aufgebaut, daß zu seinem Studium das Verständnis von Teil 2 nicht unbedingt erforderlich ist.

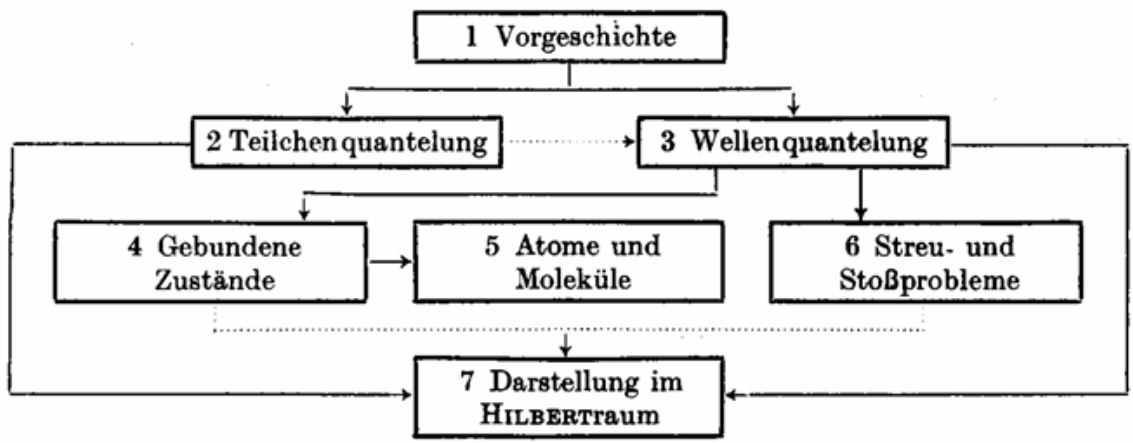

Wegen der in der Einleitung erwähnten Symmetrie der Quantentheorie gegenüber den Begriffen „Teilchen“" und „Wellen“ sollte das vorstehende Pfeilschema eigentlich ,,rechts-links-symmetrisch" sein. Dies ist aber nicht der Fall, weil sich der Wellenbegriff wegen seiner starken Anschaulichkeit zur Lösung spezieller quantenmechanischer Probleme als fruchtbarer erwiesen hat.

Geschrieben wurde das Buch in erster Linie für den lernenden Theoretiker und experimentierenden Physiker, aber auch für den theoretisch interessierten Ingenieur und Techniker, der sich über Herkunft und Grundlagen der von ihm verwendeten Formeln informieren möchte. Im übrigen ist hinsichtlich der Darstellung und Übersichtlichkeit alles versucht, um das Werk auch dem Fachkollegen, der es nicht systematisch durcharbeitet, sondern an beliebiger Stelle aufschlägt, so zugängig wie möglich zu machen. Für die Darlegung des Inhaltes galt der Grundsatz, lieber eine Erklärung zuviel als zuwenig zu geben. Trotzdem liegt es in der Natur des behandelten Stoffes, daß sein Inhalt nur dem aktiv mitarbeitenden Leser verständlich wird, der mit Bleistift und Papier die mathematisch formulierten Gedankengänge selbst verfolgt. 
Konsequent wird in allen Gleichungen das internationale Maßsystem verwendet, aber gleichzeitig durch eine geeignete Schreibweise dafür gesorgt, daß die Gleichungen von den in der Atomtheorie gewohnten Formelbildern „optisch" nicht abweichen. Gelegentliche Hinweise auf den Band ,Wellen“" werden kurt mit , W“ bezeichnet.

Das Manuskript ist in langjähriger Tätigkeit des Unterzeichneten aus Vorlesungen sowie aus dem ständigen Kontakt mit dem Lernenden und seiner Kritik hervorgegangen. Bei der endgültigen Zusammenstellung des Manuskriptes leistete Herr P. Ziesche durch wertvolle Anregungen und Kritiken in unermüdlicher Tätigkeit wichtige Hilfe, für die ihm an dieser Stelle sehr herzlich gedankt sei. Unter seiner Leitung und Verantwortung wurden auch die Kórrekturen von einem Mitarbeiterkollektiv in dankenswerter Weise durchgeführt, das sich aus den Herren G. Bessner, H. J. Kaiser, W. Kolbe, R. Lenk, D. Netzband, P. Rennert, K. Schmidt und H. J. Six zusammensetzte. Herrn B. Pegel danke ich für die sorgfältige Anfertigung der Abbildungen. Dem Verlag sei für sein verständnisvolles Entgegenkommen gedankt, mit dem er bereitwillig auf alle Wünsche des Verfassers eingegangen ist.

Dresden, den 10. Februar 1959

Wilhelm Macke

\section{VORWORT ZUR ZWEITEN UND DRITTEN AUFLAGE}

Die erste Auflage war unvorhergesehen rasch vergriffen. Daher standen Autor und Verlag vor der Aufgabe, kurzfristig eine zweite und nunmehr auch eine dritte Auflage vorzubereiten, die hiermit vorliegt. Änderungen gegenüber der ersten und der zweiten Auflage wurden nur in kleinerem Umfang vorgenommen. Insbesondere konnten alle inzwischen bekannt gewordenen Druckfehler berichtigt werden. 